\title{
Physical Parameters of 59 Stars with Planets
}

\author{
G. Cayrel de Strobel \\ Observatoire de Paris-Meudon, 5 place Jules Janssen \\ F92195 Meudon cedex France
}

\begin{abstract}
Astrometric, kinematic, spectroscopic and internal stellar structure data of 59 out of 102, extra-solar planet stars (thereafter ESPs), as yet discovered, are herewith discussed. The remaining 43 have been discarded, because they are not yet subjected to detailed spectroscopic analyses. The assembling of true physical parameters of ESPs has been made with the help of the Hipparcos and the $[\mathrm{Fe} / \mathrm{H}]$ Catalogues.The retained 59 ESPs , could be divided into three sub-samples: midly metal poor, metal normal and metal enriched ESPs. Three observational $\left(\log T_{\text {eff }}, M_{b o l}\right)$ diagrams for each of the metallicity groups have been constructed and interpreted with the help of theoretical HR diagrams, computed for metalpoor, metal-normal, and metal-rich internal structure models. The age of 47 evolved ESPs of the sample has been estimated from the relevant isochrones. Even if the age difference between some of the ESPs is great, (from 1 to $>12$ Gyr) stars as young as the Hyades (670 Myr) have not been found. ESPs are biased towards metallicities higher than solar. In this sample the number of ESPs with metallicity $[\mathrm{Fe} / \mathrm{H}]>0$ is 2.4 times the number of ESPs with $[\mathrm{Fe} / \mathrm{H}]<0$, whereas the proportion is roughly inverted in a sample of non ESPs.
\end{abstract}

\section{Introduction}

The author of this paper is not involved in the discovery of extra solar planets, but is very much interested in their parent stars, and thinks that a comprehensive study of ESPs is still worthwhile today, in spite of their robust literature. The parent stars can furnish precious informations on the kinematics, chemical composition, physical structure, degrees of evolution and, consequently, ages of the observed planetary systems. For example how great is the spread of the space velocities, chemical composition, and age, for these stars? Are we allowed (Cayrel de Strobel et al. 1981; Cayrel de Strobel et al. 1989; Friel et al. 1993), to call most of them solar analogs?

The 102 ESPs, (Schneider 2000) known at the epoch of our IAU Symposium 219 (July 2003), are all contained in the Hipparcos Catalogue, (ESA 1997), whereas only 59 ESPs where found in the edition 2001 of the $[\mathrm{Fe} / \mathrm{H}] \mathrm{Catalogue}$ (Cayrel de Strobel and Soubiran 2001), and in some recently published papers, as: (Gonzalez et al 2001; Santos et al 2003). This signifies that only a little more than $50 \%$ of the already discovered ESPs have effective temperature, spectroscopic gravity, chemical composition determined by means of detailed spectro- 

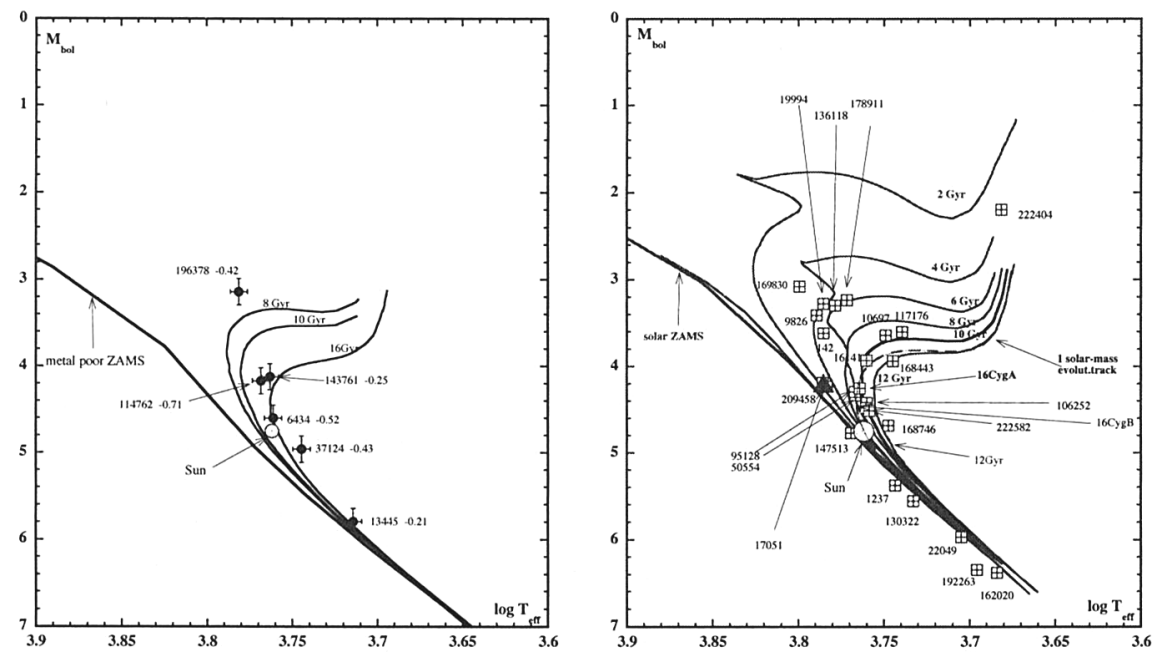

Figure 1. Left: HR diagram of 6 metal-poor ESPs. Right: HR diagram of 26 ESPs of solar metallicity.

scopic analyses, whereas the distances of the 102 ESPs are all well known, thanks to Hipparcos. A good number of the 59 ESPs have been analyzed in detail more than once. In the following, we only concentrate on these 59 ESPs, because, even if their number is not very large, these stars were not only stars with planets, but have reliable atmospheric parameters. Sect. 2 discusses three observational HR $\left(\log T_{e f f}, M_{b o l}\right)$, diagrams, obtained for metal-poor $([\mathrm{Fe} / \mathrm{H}]<-0.15)$, metalnormal $(-0.15<[\mathrm{Fe} / \mathrm{H}]<0.15)$, and metal-rich $([\mathrm{Fe} / \mathrm{H}]>0.15)$ stars of the sample. These diagrams were compared to 3 theoretical $\left(\log T_{\text {eff }}, M_{b o l}\right)$ diagrams computed with a grid of metal-poor, metal-normal, and metal-rich models with the CESAM code (Lebreton 2000). The interest in using the CESAM code is that, in it, the Sun is the calibration star and becomes the exact zero point for its comparison with the program stars.

In sect. 3 the statistical distributions of the physical parameters of the program ESPs, are shown and discussed, and sect. 4 gives the conclusions.

\section{Three $\left(\log T_{e f f}, M_{b o l}\right)$, HR diagrams for 59 metal-poor, metal-normal, and metal-rich ESPs}

We present in Figs 1 and 2 the observational and theoretical $\left(\log T_{e f f}, M_{b o l}\right)$ diagrams. As already mentioned, we have used Hipparcos parallaxes (ESA 1997) as the basis for $\left(M_{b o l}\right)$ of the program stars. The bolometric corrections where those of Alonso,taking into account metallicity and gravity effects (Alonso et al. 1996). The effective temperatures were taken from the $[\mathrm{Fe} / \mathrm{H}]$ Catalogue (Cayrel de Strobel and Soubiran 2001) and from two recent papers by (Gonzalez et al 2001; Santos et al 2003). In Fig.1a the observational $\left(\log T_{e f f}, M_{b o l}\right)$ diagram is not very crowded: only 6 metal deficient ESPs compose it. The position of these stars are compared to a metal poor ZAMS and three isochrones computed 


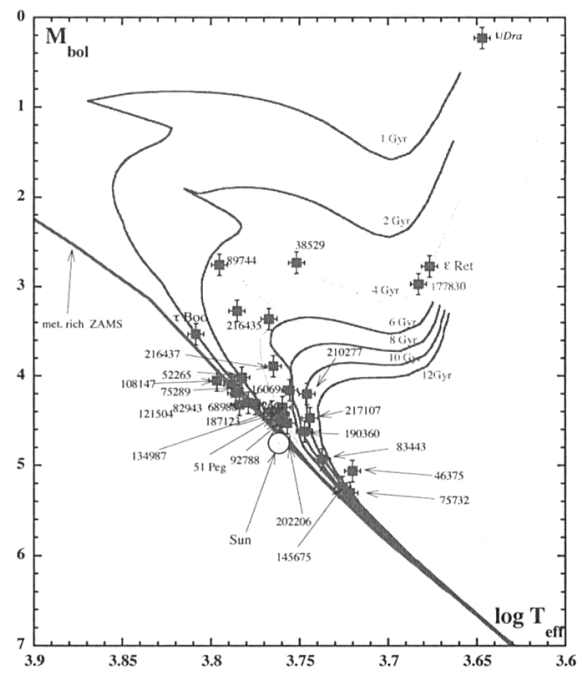

Figure 2. HR diagram for 27 metal-rich ESPs.

for $8,10,16, \mathrm{Gyr}$ for: $\mathrm{Y}=0.256, \mathrm{Z}=0.0104, \mathrm{l} / \mathrm{H}_{p}=1.64$. The ages of these metal-poor ESPs bracket between $6 \mathrm{Gyr}$ and more than $12 \mathrm{Gyr}$. Figs.1b and 2 show respectively the observational $\mathrm{HR}$ diagrams for metal normal and metalrich stars, compared to metal-normal and metal-rich theoretical ZAMSs and isochrones. These two diagrams are much more populated. Clearly we see in Fig.1b that there is a lack of metal normal ESPs between 6 and $10 \mathrm{Gyr}$, maybe indicating two bursts of ESPs in two periods: between 4 and 6 and 8 and 12 Gyr. The metal-rich ESPs populate their isochrones in Fig. 2 more uniformly between 1 and 12 Gyr. A more detailed discussion of these diagrams and new distributions of other interesting parameters of ESPs will soon appear in A\&A.

3. Distributions of the atmospheric parameters: Teff, $[\mathrm{Fe} / \mathrm{H}]$, age, and of the $V$ velocity component of the analysed ESPs

For lack of space we show here only the distributions of the four main parameters involved in our study. Histograms of other parameters as $M_{b o l}$ will be found in the A\&A paper. Figs 3 represent the histograms of $T_{\text {eff }},[\mathrm{Fe} / \mathrm{H}]$, age, $\mathrm{V}$ component (in the direction of galactic rotation), of the space velocity. The $T_{\text {eff }}$ is practically peaked at the solar value. On the contrary, the metallicity histogram is strongly asymmetric with 2.4 times more stars over solar metallicity than below. The most interesting histogram is the age one.It is rather complex, suggesting a bimodal distribution with a maximum near $4 \mathrm{Gyr}$, and another one near $10 \mathrm{Gyr}$, probably induced by a large proportion of metal-rich ESPs. The rotational lag visible in the $\mathrm{V}$ histogram is well known to exist in the old disk stars. 

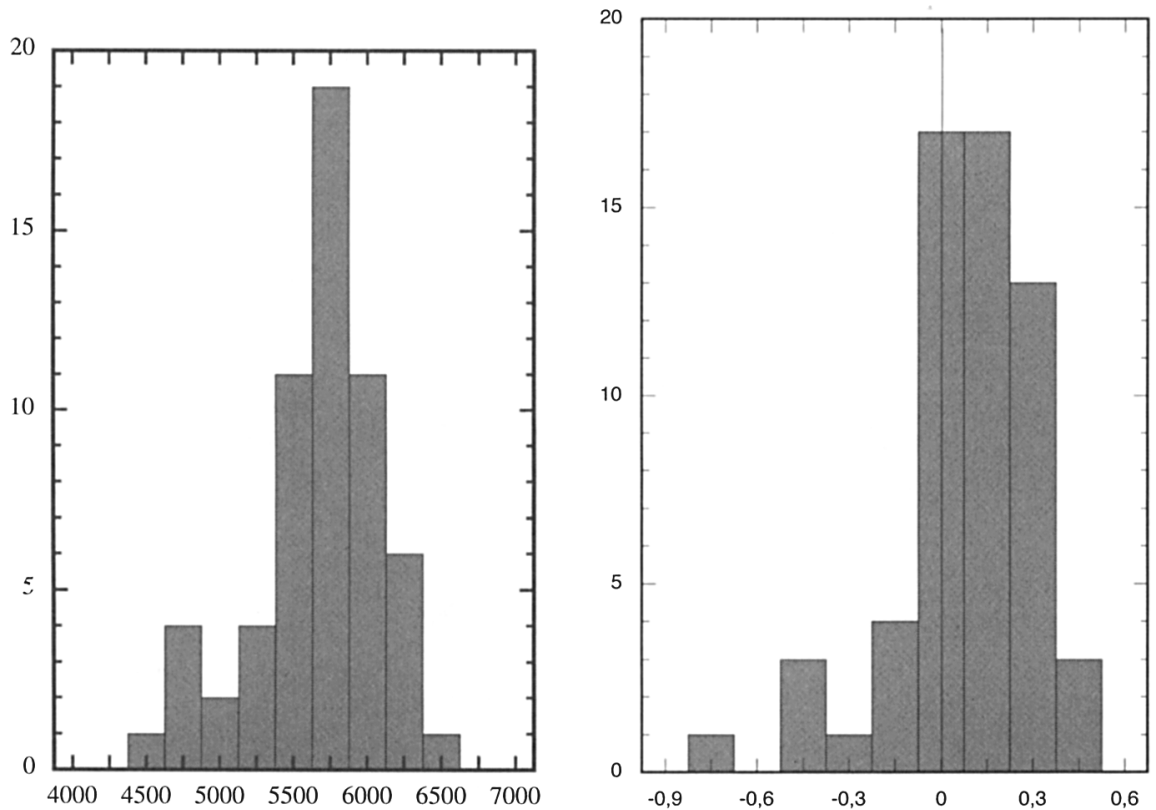

Figure 3. Left panel:histogram of $T_{\text {eff }}$ for 59 ESPs. Right panel: histogram of $[\mathrm{Fe} / \mathrm{H}]$ for the analysed ESPs. See text for comments
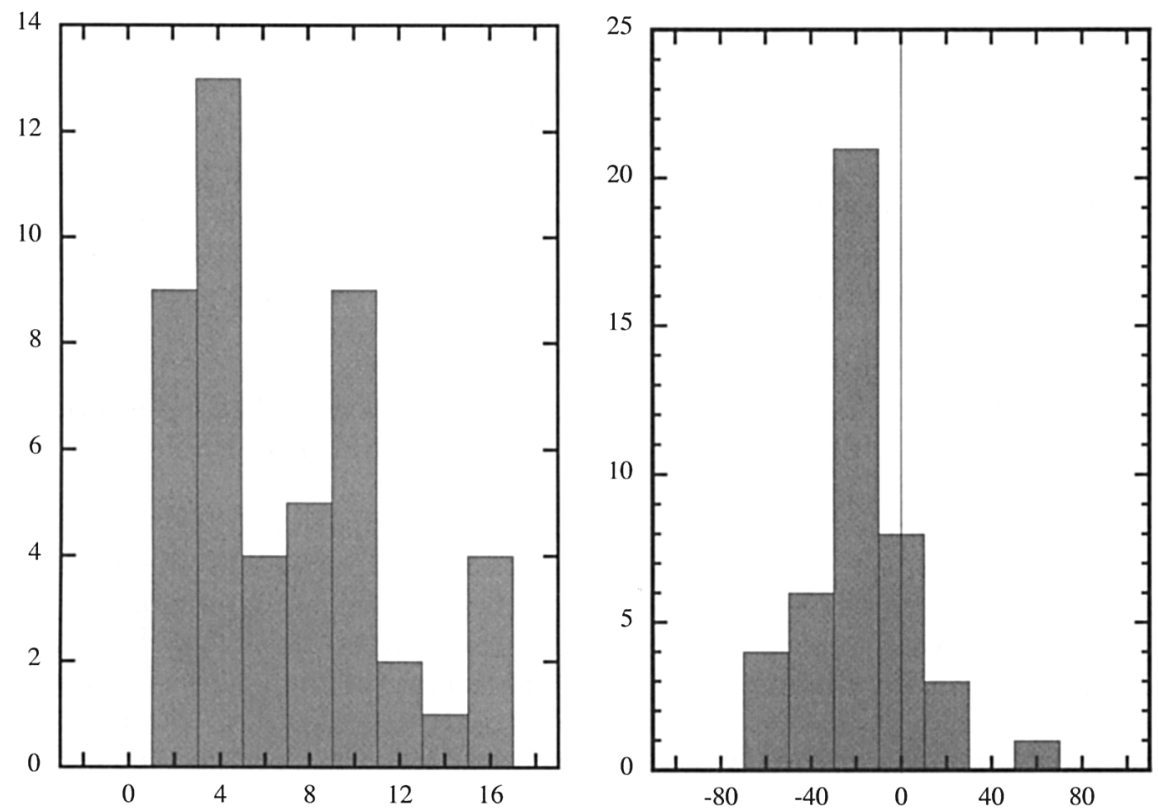

Figure 4. Left panel: histogram of the age of 47 slightly evolved ESPs. Right panel: histogram of the space velocity component V of 43 ESPs 


\section{Concluding remarks}

- ESPs have $T_{\text {eff }}$ and $M_{\text {bol }}$ strongly peaked around solar values.

- The asymmetry of the metallicity histogram is 2.4 to 1 in favor of stars more metal-rich with respect to less metal-rich than the Sun, whereas this proportion is inverted in non ESPs.

- Frequency of ESPS in the thick-disk is unfortunately still unknown because of too small statistics.

- The age distribution of EPSs is bimodal, reflecting the mean age of the thin disk and the presence of an old metal-rich population.

Acknowledgments. I thank Andrea Dupree and Arnold Benz for having made it possible to deliver this contribution to IAU Symposium 219.

\section{References}

Alonso, A., Arribas, S., \& Martinez-Roger, C. 1996, A\& A, 313, 873

Cayrel de Strobel, G., Soubiran, C. \& Ralite,N. 2001, 373,159

Cayrel de Strobel, G. et al., 1981, A\& A 94,1

Cayrel de Strobel, G., \& Bentolila, C., 1989, A\& A, 211,324

ESA 1997, The Hipparcos and Tycho Catalogues ESA SP-1200, Noordwijk:ESA

Friel, E.D., Cayrel de Strobel, G., Chmiielewski,et al., 1993, A\& A, 274, 825

Gonzalez, G., Laws, C., Tyagi, S., \& Reddy E., 2001, AJ 121, 432

Lebreton, Y.2000 ARA\& A 38,35

Santos, N.C., Israelian, G. Mayor, M. Rebolo, R. \& Udry, S. 2003, A \& A, 398, 376

Schneider, J. 2000, "Extrasolar Planets Encyclopedia", http://www.obspm.fr/encycl.html 